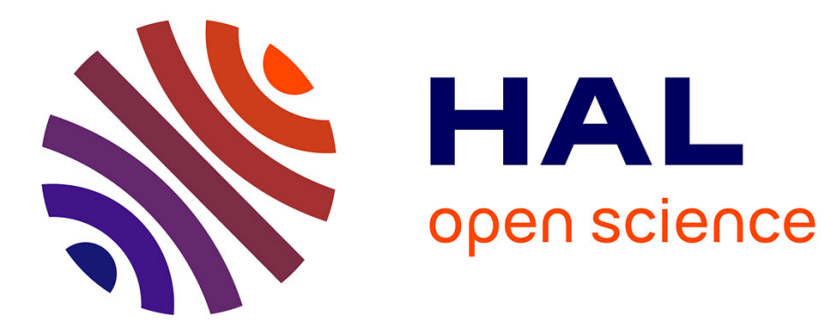

\title{
Les monnaies gauloises du musée Carnavalet
}

\author{
Brigitte Fischer
}

\section{To cite this version:}

Brigitte Fischer. Les monnaies gauloises du musée Carnavalet. Gallia - Fouilles et monuments archéologiques en France métropolitaine, 1981, 39 (2), pp.181-194. 10.3406/galia.1981.1827 . hal01940679

\section{HAL Id: hal-01940679 \\ https://hal.science/hal-01940679}

Submitted on 27 Feb 2020

HAL is a multi-disciplinary open access archive for the deposit and dissemination of scientific research documents, whether they are published or not. The documents may come from teaching and research institutions in France or abroad, or from public or private research centers.
L'archive ouverte pluridisciplinaire HAL, est destinée au dépôt et à la diffusion de documents scientifiques de niveau recherche, publiés ou non, émanant des établissements d'enseignement et de recherche français ou étrangers, des laboratoires publics ou privés.

\section{(이) $\$$}

Distributed under a Creative Commons Attribution - NonCommercial - NoDerivatives| 4.0 


\title{
LES MONNAIES GAULOISES DU MUSÉE CARNAVALET
}

\author{
par Brigitte FISCHER
}

Parmi les riches collections du Musée Carnavalet' figurent vingt et une monnaies gauloises : six sont en or, quinze en bronze frappé ou coulé. Les pièces de métal précieux se répartissent ainsi : deux statères et un quart de statère des Parisii, un tiers de statère appartenant à la série dite "homotypique des Parisii" et deux quarts de statère : l'un est vénète, l'autre, caractérisé par une "branche d'aulne ", qui barre le visage, a dù ètre émis en Gaule Centrale, peut-ètre chez les Arverni. Nous ne connaissons malheurensement pas III 'origine de ces espèces, qui sont inédites. Les pièces de bronze identifiables ont été émisess par des peuples voisins des Parisii, à l'exception des potins "à la tête diabolique ", qui sont d'origine turone, mais ont connu une large diffusion². Pour ces modestes espèces, deux provenances sont connues : la monnaie portant le $n^{0}$ 259) au Musée, qui est attribuée aux Bellovaci ou aux Suessiones, a été recueillie place Jussieu en 1931. Les pièces nos 262 et 263, deux potins "à la tète diabolique», proviennent de la Caserne de la Cité. Trois monnaies au moins, au Musée Carnavalet, sont donc d'origine parisienne.

Par ailleurs, les espèces parisiaques en or ont été recueillies sur le territoire des Parisii ou chez des peuples frontaliers ${ }^{3}$. Nous ne connaissons, pour notre part, qu'une trouvaille récente faisant exception à cette constatation : un statère d'or de classe II, proche de l'exemplaire BN 7782 et pesant 7,265 g, a été découvert à Hesdin-l'Abbé (Pas-de-Calais) en 19794 . Il n'est donc pas téméraire de penser que les trois monnaies d'or des Parisii et

1 Cous avons été encouragie dans ce travail par .I. P.-M. Duval et le Dr J.-B. Colbert de Beaulien, qui a bien voulu nous aider de ses conseils. Celle élude n’aurait pas éle possible sans l'aide aussi eflicace que généreuse de M. B. de Montgolfier, Conservateur en chef du Mlusée Carnavalet el de .I. P. Périn, Conservateur : à Lous nous tenons à exprimer notre vive gratitude. Les photographies, réalisées au Département archéologicque du Musée, sont l'cuvre de Françoise Rivière, à qui nous adressons nos sinceires remerciements. M. P.-M. Duval a exécuté les reconstitutions graphiques fig. $1 \mathrm{~h}, 2 \mathrm{~b}, 3 \mathrm{~b}$ et les dessins fig. 8 et 10 , dont nous le remercions vivement.

2 J.-B. Conbrt ne Braulinc, Les potins dits "à la lele diabolique", un monnaygage indigine de la Gaule en l'oie de romanisation, dans Revue belge de numismalique, 116, 1970, p. 97-123.

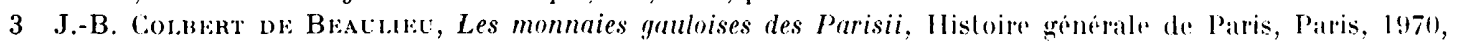
p. 63-64 ; désormais abrégé en CB, Parisii.

4 P. I.chereq, Un statere des Parisii troulé dans le Boulonnais, dans Bullelin de la sucielé frangaise de numismalique, 34, octobre 1979 , p. 574-575, fig. 1 . 

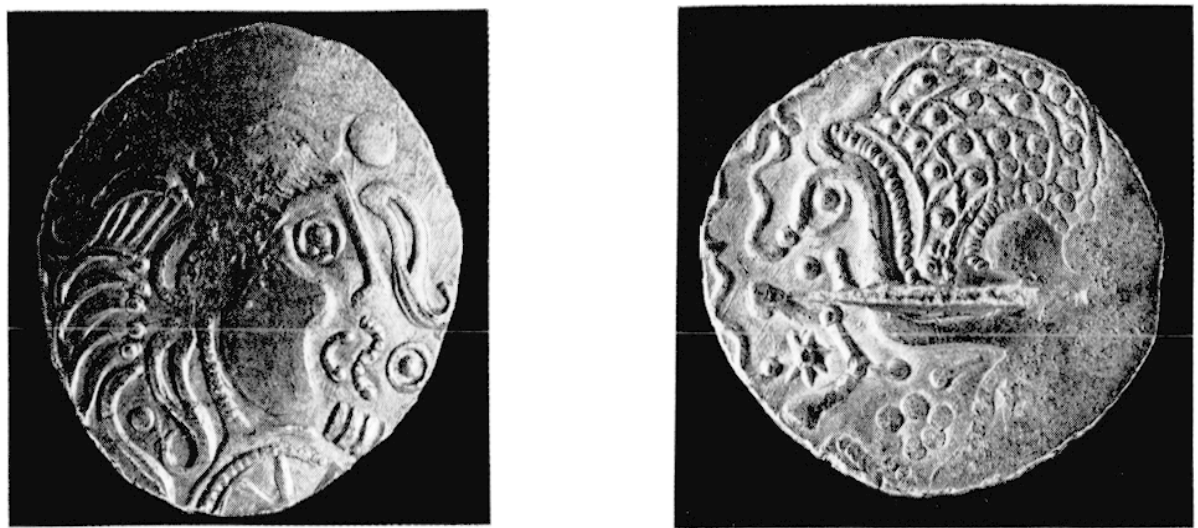

1 a No 1. Statère d'or des Parisii. Classe VI (coins D3/124). Musée Carmavalet. 7, $19 \mathrm{gr}$.
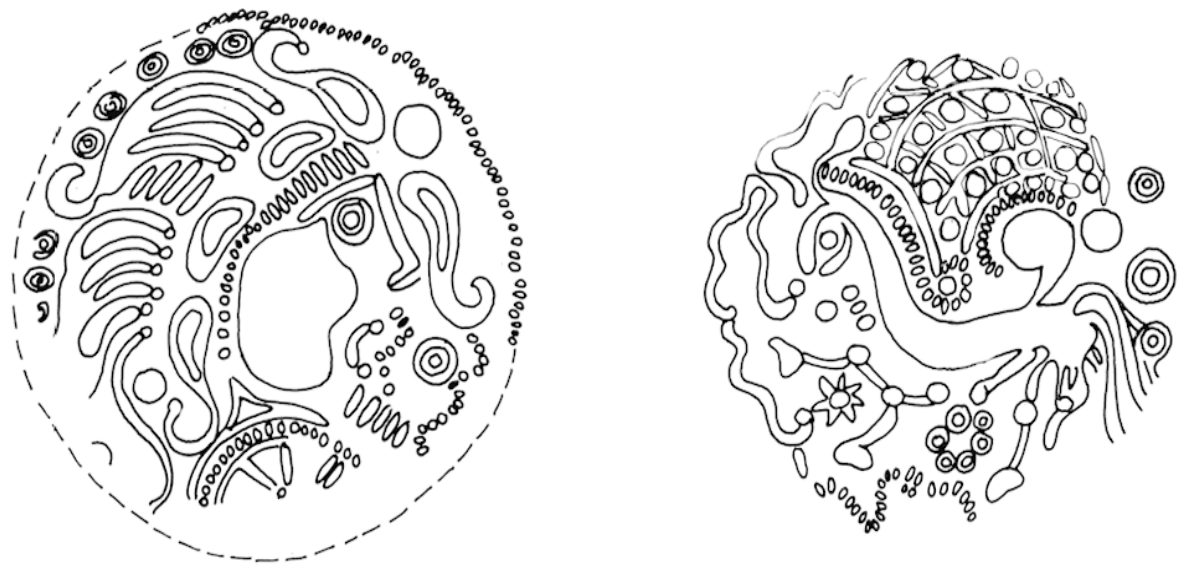

1 b Reconstilution graphique du $n^{\circ} 1$ à l'aide de 4 pièces de mèmes coins. (Iessin P.-.Y. Inval).

celle qui appartient à la série dite "homotypique" ont de fortes chances d'être d'origine locale.

Monnaies d'or (sans numéro d'inventaire) (fig. 1 a).

1. Statère des Parisii (fign. 1 a), présentant au droit un visage humain, à droite, au rendu assez désarticulé. L'œil est fait d'un annelet centré. La chrvelure est constituèe de deux groupes de mèches qui se rejoignent en une boucle et sont séparées par quatre stries parallèles. Deux croissants creux et, en bas, une mèche en esse partiellement creuse, à l'arrière de laquelle on voit un point, séparent cet ensemble du reste du visage. Au-dessus du front, le décor mal venu à la frappe n'est pas reconnaissable. Lin bas à gauche, une grande esse dont la partie inférieure manque. Ces motifs sont des mèches ou des boucles stylisées, mais l'étude des autres images du mème coin permet de le restituer ( fig. 1 b).

Le visage est cerné vers l'arrière par un cordon perlé qui suit le contour de la mâchoire

N.B. -.- Toutes les monnaies illustrant ced arlicle sont agrandies an double. Ies pholographies 1 a, 2 a, 3 a, 4 , 6, 7 sont de F. Rivière, Musée Carnavalet. 
et s'arrète en bas sur une courbe qui représente schématiquement le cou. Derant le front, se trouve un gros cercle plein suivi d'une longue mèche en esse partiellement creuse, qui déroule son tracé harmonieux devant le nez. A l'avant de la bouche, représentée par un demi-cercle pointé, on peut voir une esse perlée et, en avant, un gros annelet centré. En dessous se trouve un motif composé de quatre courtes lignes, verticales et parallèles. Sous la coupe du cou, un fragment de roue dont le pourtour est perlé et dont trois rayons sont visibles.

Au revers, cheral à gauche, surmonté d'une sorte de grand filet dont les mailles sont pointées. La crinière est faite d'une ligne perlée que l'on retrouve autour de la grosse volute (ici écrasée) qui surmonte la croupe. Cette face porte une entaille profonde en son milieu; elle est parallèle au corps du (heval. I)u front part une ligne sinueuse, un peu informe, non identifiable. Sur la tête, une sorte de panache. Ln lien ondulé part de l'extrémité de la bouche, monte plus haut que le front ef redescend jusqu'entre les antérieurs. Entre les jambes avant, qui sont réunies au départ en un seul jambage, une étoile à sept branches est visible, placée à l'extrémité du lien, qui est pointé. Le cheval est sexué. Sous le ventre, une rosace. Au-dessous, un lien perlé sinueux.

Ce statère appartient à la classe VI déterminée par J.-B. Colbert de Beaulieu ${ }^{5}$, pour laquelle quatre coins de droit et cinq de revers sont connus. Il pèse $7,19 \mathrm{~g}$ et a un module de $2: 2 / 24 \mathrm{~mm}$. Il est issu du même coin de droit (VI D 3) et de revers (VI R 4) qu'une monnaie conservée au British Museumbi . Cette dernière pièce porte curieusement une entaille identique à celle qui se trouve sur le statère du Musée Carnavalet, mais elle est située juste au-dessus du corps de l'animal. Un autre statère figurant dans la collection Vian, à Avignon, est également issu dess mèmes coins de droit et de revers? la monnaie BN 77798. Quatre pièces des mèmes coins permettent donc de faire une reconstitution graphique de l'image presque entière (fig. 1 b). La pièce de la collection Vian d'A vignon complète le droit au-dessus de la tete, alors que celle du British Museum permet, pour le revers, de reconstituer l'arrière-train du cheval, dont le dessin est écrasé sur l'exemplaire du Musée Carnavalet. La monnaie BN 7779) complete le lien perlé, sinueux, qui se déroule sous la rosace.

2. Statère d'or des Parisii (fig. 2 a), orné au droit d'une tète humaine à droite. La chevelure est constituée de mèches terminées par des spirales et réunies parfois par un petit trait. Entre ces mèches et le visage, on distingue deux croissants et une longue esse en partie creuse. Une autre esse creuse se déroule devant le visage, mais elle n'est pas visible en totalité. Ces motifs sont des mèches ou des boucles stylisées. L'œil est punctiforme, le nez très droit, les lèvres sont un demi-cercle bouleté relié par un trait vertical à la partie inférieure du nez. La coupe du cou est suivie, en bas, de stries verticales, parallèles. Sous le menton, on voit, écrasé, une sorte de fuseau hérissé, qui est connu dans ce monnayage.

Le revers présente un cheval stylisé, à gauche. Au-dessus de son dos se développe une sorte de filet pointé. La crinière est figurée par une longue ligne perlée, qui se termine en spirale au milieu du dos. Au-dessus de la croupe, une forte spirale, semblable à celle de la monnaie précédente. Entre la jambe gauche et la queue, un trait sinueux. In lien onduleux part de la bouche de l'animal. Au-dessus du front est l’amoree du panache. Le cheval n'est 

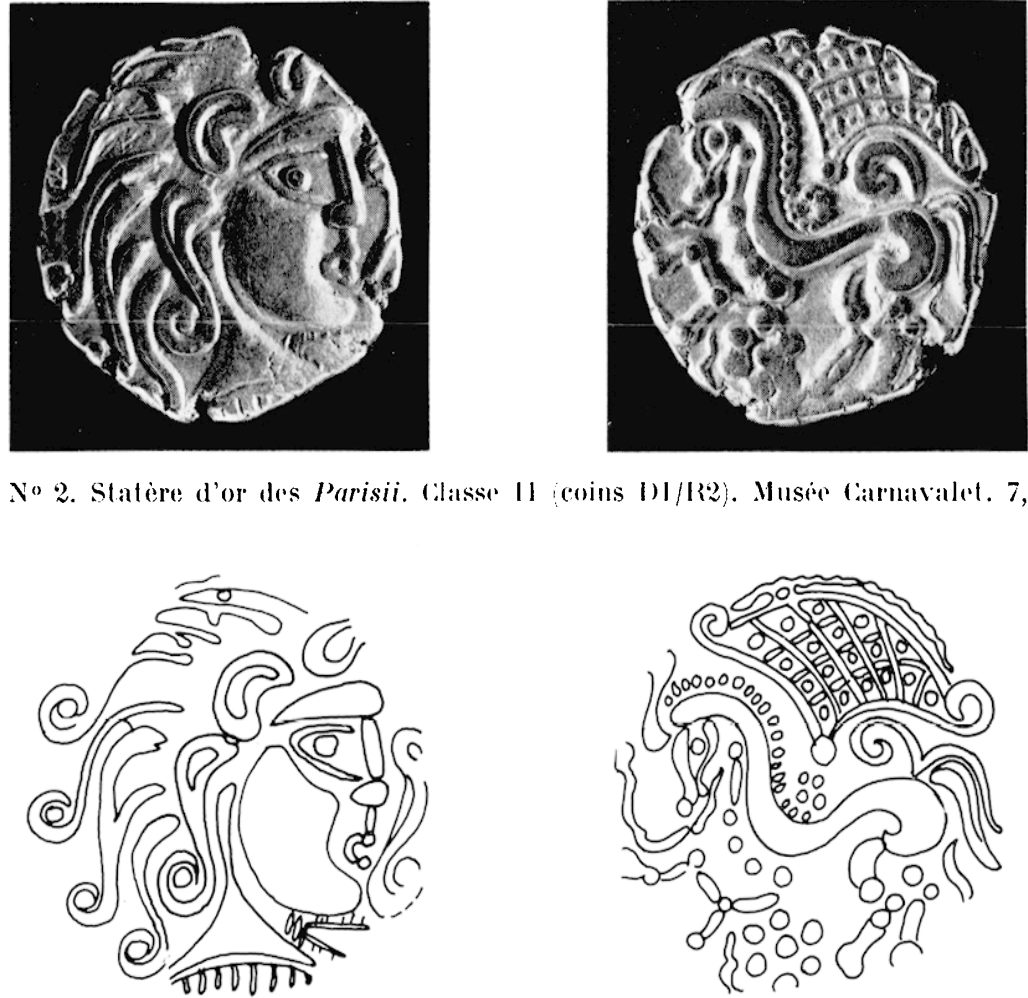

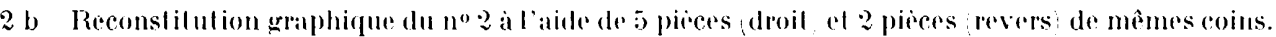
(I)essin de I'.-.M. Dumal).

pas sexué. Cing globules ornent le devant du poitrail ; sous les jambes avant, unies au départ, on voit une rosace, constituéc de sept globules, à gauche de laquelle est l'extrémité d'un lien souple et vertical, dont le pendant existe sous l'arrière-train de l'animal.

Cette monnaie appartient a la classe 11 de J.-B. Colbert de Beaulie ${ }^{9}$, pour laquelle quatre coins de droit et quatre de revers sont connus. Elle pise $7,21 \mathrm{~g}$ pour un module de $20 \mathrm{~mm}$. Elle est issue du mème coin de droit que les quatre pièces suivantes : BN 7782, vente Lockett 1955, vente Vinchon-Ciani 195) et vente Vinchon 1963 $3^{10}$ et du mème coin de revers que BN 778211. Elle est, done, selon le classement étahli par J.-B. Colbert de Beaulieu, issue des coins II I)1 et II R2. Ces esperese, cing pour le droit el deux pour le revers, se completent, puisque l'exemplaire de la vente Vinchon 19633 fournit un pelit motif en forme de croissant, gravé devant te front et permet de terminer le tracé de la grande esse qui se déroule devant le visage. Par ailleurs, elle donne une vue blargie des stries vertieales, sous

5) CiB, Parisii, p. 37-43.

6 Op. cil., p. 11 , fig. $26, \mathrm{n}^{\circ} 1$.

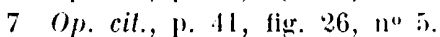

\& Op. cil., 1). 11, li⿺r. 20, 110 's.

9) Op. cil., p. 7-11.

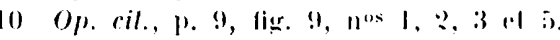

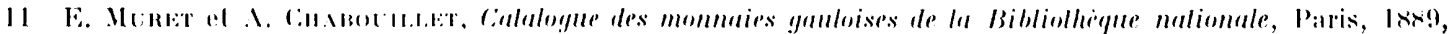
p. 179 . 

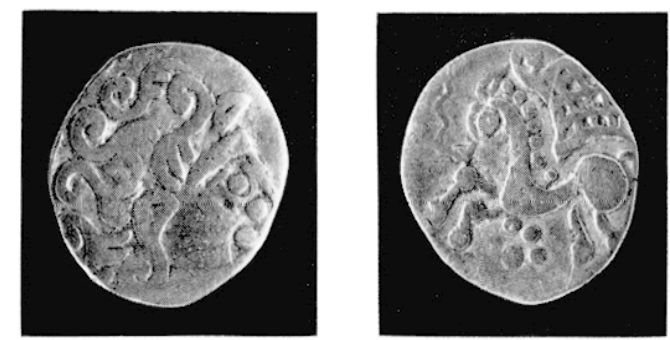

3 a No 3. Quarl de slatipre d'or des P'arisii. lalsse IV. Musse Cammavalel. 1,63 g.
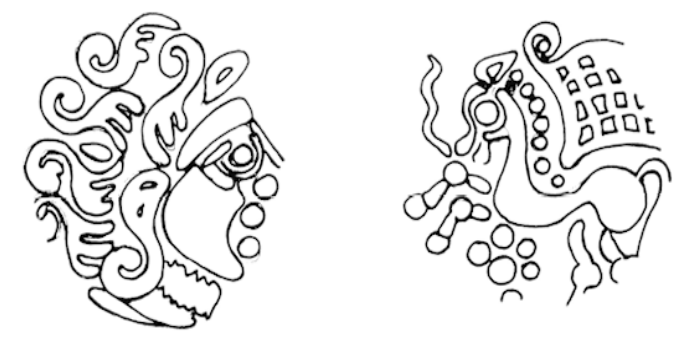

31 Reconstilution graphique du no 3 a l'aide de 2 pieces de mèmes coins. (I)essin de P'- Ml. Inural).

la coupe du cou. La pièce BN 7782 est la seule qui comporte les extrémités des quatre mèches de la chevelure, à l'arrière de la tète. Pour le revers, elle autorise la reconstitution totale du filet gravé au-dessus du cheval et apporte une meilleure connaissane du lien qui se déroule derant sa tite (fig. ? h).

3. Quart de statere d'or des Parisii (fig. :3 a). Le droit présente une tète d'homme tournée à droite. La chevelure est faite de grosses mèches de trois éléments se terminant par des spirales, qui dessinent une sorte de lyre au centre, contenant un élénent ovale au milieu. Lne esse an partie creuse est derrière la mâchoire. L'épaisse ligne des sourcils est prolongée à angle droit par la ligne du nez, très droite, terminée par un gros point ; un autre gros point figure la lìresupérieure.

Le revers est orné d'un cheval au galop, à gauche. surmonté d'une sorte de grand filet quadrillé, non pointé, dont l'extrémité gauche se termine par une spirale. Un lien sinueux part de la bouche de l'animal, une grande oreille domine le front. La crinière est faite d'une succession de points, les articulations des jambes arant sont bouletés, sous le ventre, on apercoit une rosace perlée, incomplètement venue. Le cheval n'est pas sexué.

Cette pièce appartient à la classe IV déterminée par J.-B. Colbert de Beaulieu ${ }^{12}$. Elle est de même coin de droit et de revers que BN 7791 ${ }^{13}$. Or aucune identité charactéroscopique n’avait été relevée jusqu à présent parmi les quarts de statere de cette classe ${ }^{14}$ : cette monnaie présente done un intérêt particulier car clle permet de compléter l'image du coin (fig. 3 b). Pour le droit. l'exemplaire du Cabinet des Médailles est plus complet que cette pièce pour le profil du visage, moins pour la chevelure; pour le revers. celle du Musée Carnavalet permet de mieux connaitre l'oreille du cheval et l'extrémité gauche du filet. Ce quart de statère pèse 1.63 g pour un module de $14 \mathrm{~mm}$.

4. Thers de statere en or (fig. 4). de la "série homotypique" attribuée aux Parisii, qui comporte un statere unique ot des divisions nombreuses ${ }^{15}$. Au droit, visage à droite. La cherelure est constituée de trois grandes mèches an virgules, orientées de façon différente,

$1:$ op. rit. 1. 17-:31.

13 op. cil., p. 1s, lig. 11, 1" III.

1.1 op. cil., p. 19.

15) Op. cil., p. 1-41-1511. 

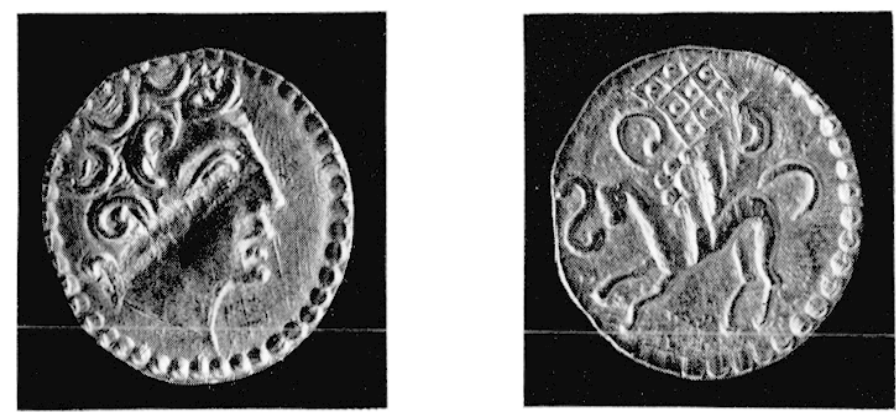

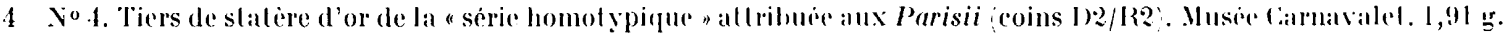

qui sont surmontées de trois boucles semi-circulaires, tournées vers l'extérieur et ornées intérieurement de petits traits parallèles. Des traits analogues se voient entre les mèches et les boucles. Le profil est régulier et harmonieux, bien moins stylisé que la chevelure. La coupe du cou est figurée par une ligne très mince et comporte une petile boucle à l'arrière. (irènetis à gros grains au pourtour. La pièce présente une large entaille oblique, qui va de l'œil à la nuque. L'image monétaire est complète.

Au revers, on voit un cheval à gauche; l'animal présente une stylisation moyenne. Au-dessus de son dos, une rosace perlée est acrostée de deux ornements semblables à de grosses virgules. L'ensemble est surmonté d'un motif quadrillé comportant neuf cases carrées et pointées; l’angle supérieur manque. l'ne grande esse à l'envers se développe devant la tète de l'animal. Cette face est également entaillée, mais verticalement. Grènetis à gros grains au pourtour, conservé pour une moitié environ.

Cette pièce est issue des mèmes coins de droit et de revers que les exemplaires BN 7803, 7804 et $7805^{16}$, ainsi qu'une monnaie conservée au Yusée de Péronne ${ }^{17}$. Elle pèse $1,91 \mathrm{~g}$, son module est de $16 \mathrm{~mm}$. Les deux images qu'elle offre sont complètes, mais abîmées par l'entaille centrale qui n'existe pas sur la pièce de P'éronne (fig. 亏). .J.-B. Colbert de Beaulieu, pour des raisons typologiques, métrologiques, technologiques et après examen des lieux de découverte, se refuse à attribuer ce numéraire aux Parisii. Il pense qu'il s'agit du monnayage d'une peuplade vraisemblablement riveraine de la Seine et située au nord-ouest de Paris $^{18}$.

4 bis ? A l'époque où .J.-B. Colbert de Braulieu a réalisé la magistrale étude de ce monnayage, il n'a pas pu avoir connaissance de ces quatre monnaies. En revanche, il a publié une pièce qui porte la mention "Musée Carnavalet" ", d'après la documentation réunie par (iabrielle Fabre, qui avait commencé l’étude de ce numéraire ${ }^{20}$. Or, ce quart de statère de classe V n'a pas été retrouvé au Musée. Ieux hypothèses peuvent expliquer

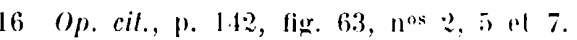

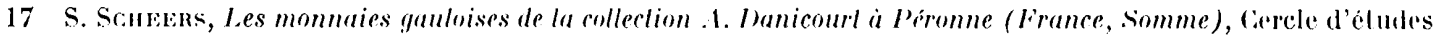
numismatiques, Traviux, Bruxielles, 1975, p. 61, " 198, p. XII, 198.

Is (:B, Parisii, p. list.

19 ()p. cil., p. 30, fier. :?1, 11" 11 .

20 Op. cil., p. 31, n. $\times 7$. 


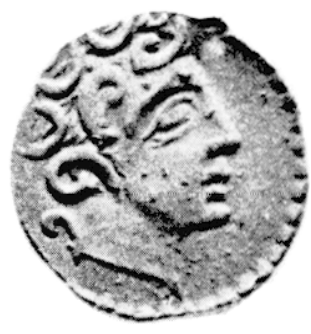

5 Tiers de statere dor de la "sirio homolypique"

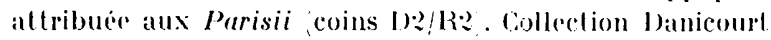
a Pirontre, 1,
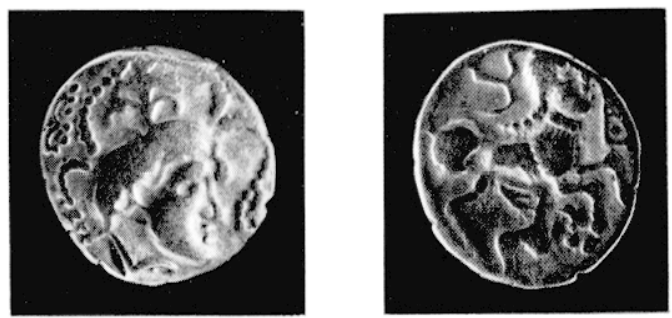

6 Quarl de slatere en or des Veneli. Nusion Ciarnamilel. $1,91 \mathrm{~g}$.

cette lacune : les réserves du Musée sont importantes et il est possible que la monnaie soit redécouverte un jour en un endroit inattendu, ou bien il s'agit d'une erreur de fiche commise par (iabrielle Fabre. Ce fait ne pourra être ni vérifié ni infirmé, l'auteur étant décédé depuis de nombreuses années.

5. Quart de statère en or (fig. 6). Le droit est orné d'une tète d'homme, tournée à droite, entourée de lignes perlées et sinueuses, deux partant du front et deux de l'occiput, en divergeant. I'apris d'autres monnaies armoricaines, ces liens se terminent chacun par une petite "tète coupée", suivant l'appellation traditionnelle. On voit d'ailleurs, en bas à droite, dans la boucle terminale du lien, un fragment de tête. Au milieu de la coiffure se dresse un motif qui évoque un petit arbre stylisé (à cet emplacement, on trouve sur certaines pièces de cette région un fleuron. Il s'agit vraisemblablement ici de ce thème décoratif, qui a été doublé verticalement). Én haut, à gauche, on peut voir une double ondulation précédéc de six gros points pratiquement alignés : s'agit-il d'une fantaisie graphique réalisée à partir des liens perlés ? En l'absence d'éléments de comparaison, il paraît difficile de trancher.

Au revers, un cheral androcéphale, à droite, occupe le champ de la pièce. Il est sanglé et porte au cou un collier perlé; la crinière se termine par quelques perles qui suivent la courbure du dos. Au-dessus de l'animal, on aperçoit une tige sinueuse tenue par un aurige, dont le corps est à peine visible sur cette pièce, mais qui est complet sur d'autres exemplaires du même type. Sous le cheval, on distingue un petit personnage ailé, couché. Devant le poitrail, parait pendre un objet indéterminable qui est soit isolé dans le champ, soit, peut-être, dépendant d'un lien qui serait tenu par l'aurige, comme l'«étendard " représenté sur de nombreuses monnaies armoricaines.

Cette monnaie est attribuable aux $V$ eneli ${ }^{21}$. Elle pèse $1,91 \mathrm{~g}$, pour un module de $12 \mathrm{~mm}$. Elle est issue des mèmes coins de droit et de revers que l'exemplaire BN $6894^{22}$, attribué au siècle dernier aux Aulerci Cenomani et centré de la même façon.

6. Quart de statère en or, présentant au droit une tète d'homme à droite (fig. 7). La chevelure est constituée de mèches serrées, l'œil est gros, il a pris une forme ovalaire,

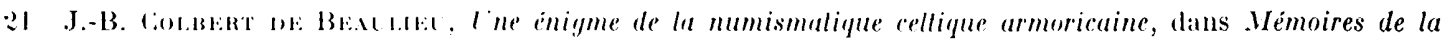
societé d'hisloire el d'archoologie de Brelagne, 34, 195.4, p. 5-38.

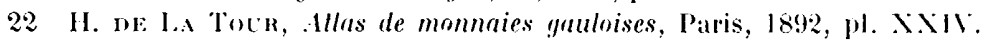



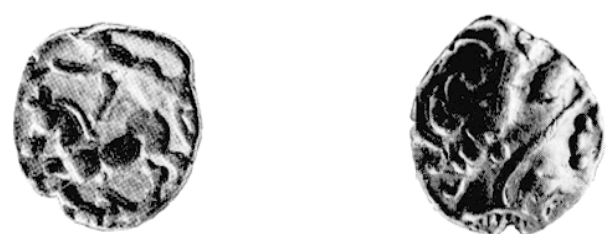

7 Ouart de slatire en or "a la branche d'aulne". Musér Carnavalel. 1,91 g.

à la suite de l'écrasement du relief. La base du nez et la bouche sont punctiformes, sous la coupe du cou, de fines stries verticales, parallèles et très courtes, limitées par un trait horizontal inférieur. Le visage est traversé obliquement par une longue ligne, que l'on a coutume d'appeler "branche d'aulne».

Au revers, un cheval à gauche, au corps assez massif, est conduit par un aurige qui tient un objet longiligne. A l'arrière de l'animal, on distingue la roue du char. La crinière est faite d'un gros trait continu, le cheval n'est pas sexué. Sous le ventre, un triscèle.

Un exemplaire du même type est conservé au Schweizerisches Landesmuseum de Zurich ${ }^{23}$, et notre pièce est proche des exemplaires BN 3663, qui comporte aussi le triscèle sous le cheval, et BN 3664 (sur cette monnaie, toutefois, l'animal est à droite), mais on ne connaît pas d'identité de coin à son sujet. Elle pèse $1,91 \mathrm{~g}$ et a un module de $11 \mathrm{~mm}$.

La pochette de papier qui contenait cette pièce portait la mention manuscrite "Quart de statère parisien. Type se rapprochant du statère des Bellovaques (Beauvais) ". Cette identification est erronée : la monnaie n'a aucun lien avec le numéraire des Parisii; il s'agit vraisemblablement d'une frappe de ciaule Centrale, peut-être arverne.

L'étude de ces six monnaies d'or impose quelques réflexions sur leur métrologic et fournit d'utiles indications chronologiques. Les trois pièces classées aux Parisii sont vraisemblablement datables dans le premier tiers du .. I er siècle ${ }^{24}$ et les émissions correspondent à l'ordre des classes déterminé par J.-B. Colbert de Beaulieu ${ }^{25}$. Le statère de la classe VI se situant probablement à la fin de cette période, vers les années -70 : une baisse nette de l'aloi apparait en effet avec la création de cette série.

Ces statères ont des poids qui correspondent à ceux des exemplaires de la même classe répertoriés par .J.-B. Colbert de Beaulieu. Il n'en est pas de même pour le quart de statère de classe IV, qui est exceptionnellement léger : le poids des divisions de ce type culmine en effet à près do $1,80 \mathrm{~g}$, la division la plus légère connue à ce jour pesait 1,67 $\mathrm{g}^{26}$. Or, le quart de statère du Musée Carnavalet pèse seulement $1,63 \mathrm{~g}$, ce qui est particulièrement bas. Une telle division correspond à une unité de $6,60 \mathrm{~g}$ environ, alors que pour cette classe, parmi les statères dont nous connaissons le poids, le moins lourd, celui de la collection SmithLesouëf, pèse $7,2622 \mathrm{~g}^{27}$.

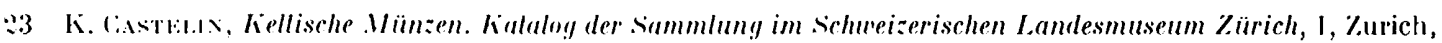
s.d. $1978 \%$, p. $15 x$, , $^{\circ} .114$.

21 CB, l'ilrisii, p. 106-10s.

25) Op. rit., p. 18-52.

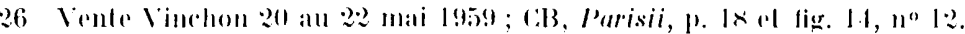

27 CB, larrisii, p. 17. 
Le tiers de statere appartenant à la série dite "homotypique des Parisii "se situe, au contraire. dans l'échelon de poids le plus courant : celui de 1,94 à $1,90 \mathrm{~g}^{28}$. L’émission des esperees de re type est datée par J.-B. Colbert de Beaulieu vers $-70^{29}$.

Quant aux deux autres quarts de statère. l'un à la branche d'aulne, émis dans un atelier de Ciaule C'entrale, l'autre d'origine vénète, ils paraissent en or de haut aloi et leur poids est élevé : $1,91 \mathrm{~g}$, ce qui correspond à une unité de 7,60 g. Il ne s'agit pas là des premières imitations du statère de Philippe II de IIacédoine, puisqu'elles pesaient entre 8,60 et $8 \mathrm{~g}^{30}$, mais de celles de la deuxième série, datable vers la fin du - $\mathrm{u}^{\mathrm{e}}$ siècle; espèces sont, de toute façon, antérieures aux monnaies des Parisii.

Monnaies de bronze inventoriées.

7. No 249 Musée. - Bronze frappé qui ne peut ètre identifié avec sûreté en raison de son mauvais état de conservation. Le droit parait proche de celui de l'exemplaire BN $7606^{31}$, attribuć aux Meldi. Le revers est dans un état tellement dégradé qu'il ne permet aucun rapprochement. Cette monnaic pèse $2,57 \mathrm{~g}$, pour un module de $14 \mathrm{~mm}$.

8. No 250) Musée. -.. Deux fragments de monnaie de potin attribuée aux Catalauni, de type BN $8124^{32}$.

9. No 253 Musée. - Bronze frappé, dont le droit est en très mauvais état. Le revers, mieux conservé, présente un cheval androcéphale au galop à droite d'un rendu assez mou ; sa crinière est perlée, un gros annelet centré se trouve sous le ventre de l'animal. En dessous, on aperçoit un motif tripartite qui ressemble à un triscèle. On distingue un gros oiseau au-dessus du cheval. Cette pièce est proche de $\mathrm{BN} 7850^{33}$. Le droit était probablement orné d'une tète humaine à gauche; devant la face devait se trouver la légende VENEKTOC: en lettres grecques. Cette série figure dans le Catalogue des monnaies gauloises de la Bibliothèque nalionale, de Vuret et Chabouillet, sous la rubrique Venextos ${ }^{34}$ et H. de La Tour ${ }^{35}$ l'attribue aux Silvanecles. Poids : 3,999 g, module : $16 \mathrm{~mm}$.

10. No 254 Musée. - Cette monnaic de bronze frappé, oxydée, est recouverte en surface d'une pellicule dorée, très brillante, qui adhère aux reliefs les plus forts. Le droit, en très mauvais état, ne peut ètre identifié. Au revers, on voit un oiseau semblable à ceux qui ornent le monnayage attribué aux Senones, de type BN 7490 à $7565^{36}$. La pièce pèse $1,86 \mathrm{~g}$. pour un module de $15 \mathrm{~mm}$.

$2 \times$ Op. cil., p. 1.45.

29 Op. cil., p. 147.

30 S. Schlers, Les imilations en Gaule du slatere de Philippe II de Macédoine, dans Internalional Numismatic symposium, Budapest Akademiai Kiadó, 1980, p. 41-53,.

31 H. ne: LA Totn, Allas.... pl. XXX.

32 (op. cil., pl. XXXil.

33 op. cil., pl. XXXI.

3.4 I'. $1 \times 1$

35) Mllas... pl. XXXI.

36 H. DE I.A Torr, Allas..., pl. XX. 

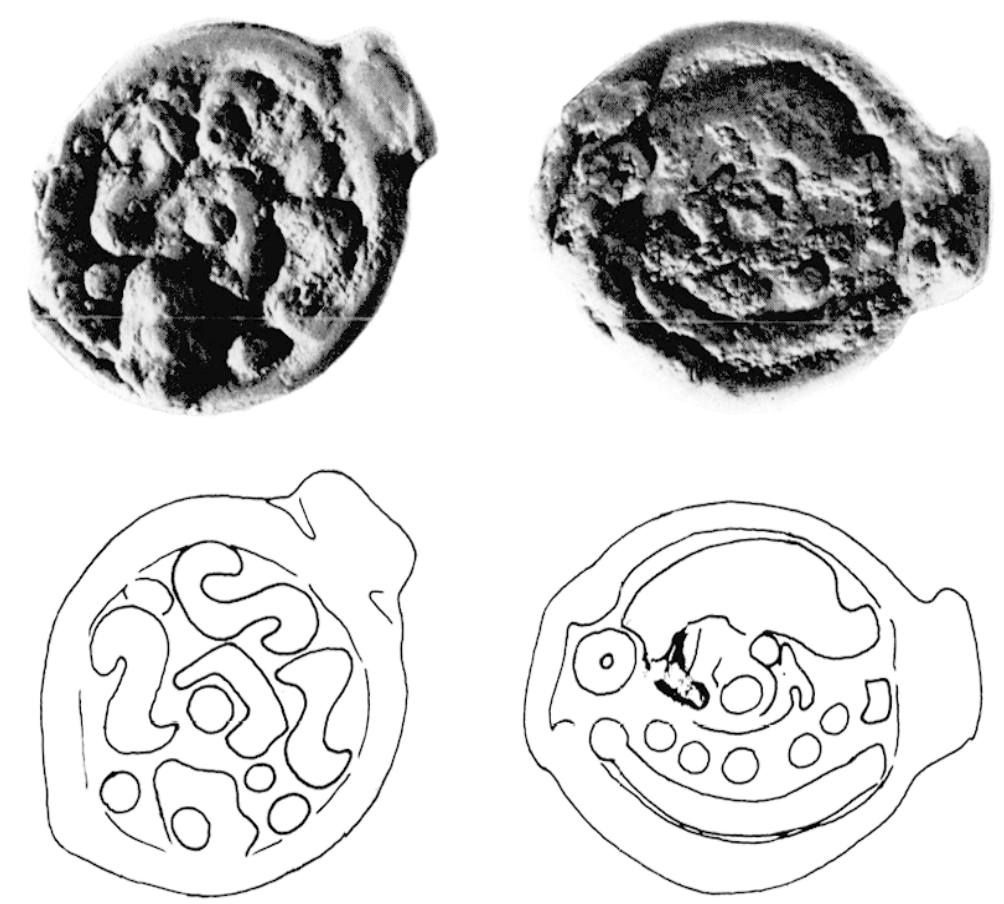

Monnaie de potin des Bellovaci ou des suessiones. Musie (alrmatalet. 1,50 g. (I)essin P.-.M. Iuval el F. Blind).

11. No 25:5 Musée. - Bronze frappé en très maurais état. Au droit, on devine un visage humain, au revers, on voit un oiseau, à gauche. Cette pièce paraît proche de l'exemplaire BN 754\%37, attribué aux Senones. Son poids est de $2,26 \mathrm{~g}$, son module de $15 \mathrm{~mm}$.

12. No 256 Muséc. - Bronze frappé, fragmentaire. Le droit présente des traces de chevelure constituée de gros traits courbes. Au revers, on aperçoit un aigle, dont le rendu, autant que l'on en puisse juger en raison du mauvais état de la pièce, paraît proche de celui de la monnaic BN $7606^{38}$. Cette série est attribuée aux Meldi.

13. No 257 Musée. - Bronze frappé, très oxydè. Le droit est si dégradé qu'il n'est pas identifiable. Au revers, on peut reconnaître un cheval au galop, à droite. Cette monnaie, autant que l'on en puisse juger en raison de son mawais ètat, parait proche de l'exemplaire BN 8440, attribué aux Ambiani. Eille pèse $3,09 \mathrm{~g}$ et a un module de $15 \mathrm{~mm}$ environ.

14. No 259 Musée. - Monnaie de polin oxydée, qui fait partie de la série conservéc à la Bibliothique nationale sous les nos 7906 a $792: 3$ ot est attribuée par L. Muret et A. Chabouillet ${ }^{39}$ aux Bellovaci (fig. 8 : photo et dessin). Sur une face, on peut voir un visage humain à droite, fortement dégénéré, composé surtout de lignes courbes et de globules. Sur l'autre, un annelet pointé occupe le centre du champ; il est surmonté d'une sorte de crinière,

37 op. ril., pl. XX..

38 op. cit., pl. XXx.

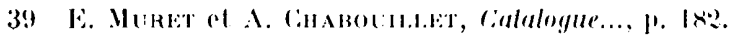




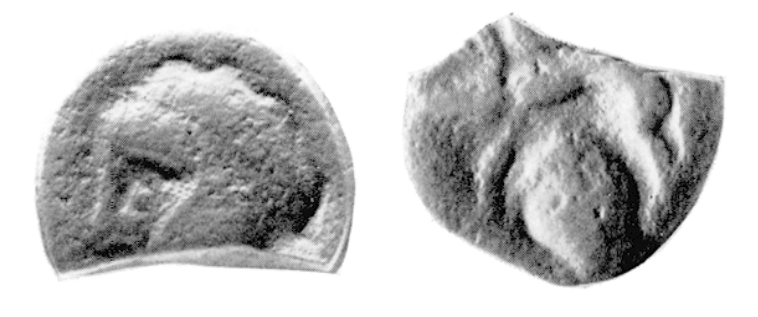

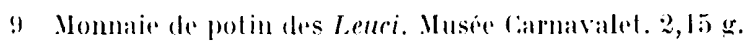

qui ast, pour les auteur's du Calalogue des monnaies gauloises de la Bibliothèque nalionale, de mème que pours. Scheers ${ }^{40}$, un sanglier : il s'agirait dans ce cas d'un animal particulièrement stylisé. Sur d'autres espèces mieux conservées, on voit qu'il est tourné à droite. In trait oblique tient lieu de tête et on peul voir un petit cercle à l'arrière. A la partie inférieure de la monnaie le bourrelet de coulée est en quelque sorte doublé par une ligne courbe bouletée à chaque extrémité, qui pourrait représenter un torque. Ce décor est surmonté de cinq gros points disposés en demi-cercle. E. Muret et A. Chabouillet attribuent ces espèces aux Bellovaci. S. Scheers les donne à ce peuple ou à celui des Sutessiones.

La pièce pèse $4.50 \mathrm{~g}$, pour un module de $20 \mathrm{~mm}$ environ. Le sachet de papier dans lequel elle est conservér au Musée porte la mention "place Jussieu - 1931 - IJon de II. Cirimault".

15. No 260 Musée. . Honnaie de potin fragmentaire (fig. 9). La partie conservée représente un peu plus de la moitié de la pièce. Au droit se trouve une tête humaine à gauche, au revers, on reconnaît un sanglier entre les pattes duquel on distingue une grosse masse ovale. Cet exemplaire est proche du no BN 918041, attribué aux Leuci. Sur cette dernière pièce le décor qui se trouve sous le cheval est parfaitement identifiable : il s'agit d'un masque humain. Ce fragment pèse $2,15 \mathrm{~g}$; module : $16 \mathrm{~mm}$.

16 et $1 \%$. Yos $262-2633$ Musée. - Il s'agit de deux monnaies de potin dites "à la tête diabolique». Sur la face qui tient lieu de droil, on devine une tete humaine, à gauche, extrêmement stylisée; l'œil et la bouche, démesurés, représentés par deux dépressions circulaires asse grandes, donnent a ce visage une expression saisissante. Sur l'autre face, on aperçoit un quadrupède à gauche, dont la queue est relournée au-dessus du dos. Ces espèces, émises en grandes séries par les Turones, sont de fabrication tardive ${ }^{42}$. Elles sont classées à la Bibliothèque nationale sous les nos 5646 à 5688 .

Ln exemplaire très proche de ceux-ci a été publié par S. Scheers ${ }^{43}$. L'une des monnaies pèse $2,88 \mathrm{~g}$, pour un module de $15 \mathrm{~mm}$; l'autre a un poids de $2,96 \mathrm{~g}$, son module est de $14 \mathrm{~mm}$ (fig. 10 : photo et dessin). La pochette qui les renferme porte la mention : "Caserne de la Cité ".

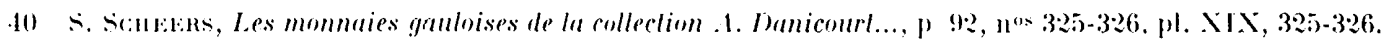

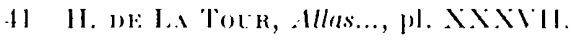

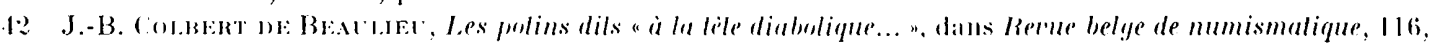
1970, p. $97-1: 3$.

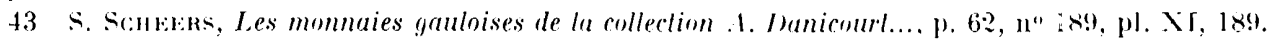



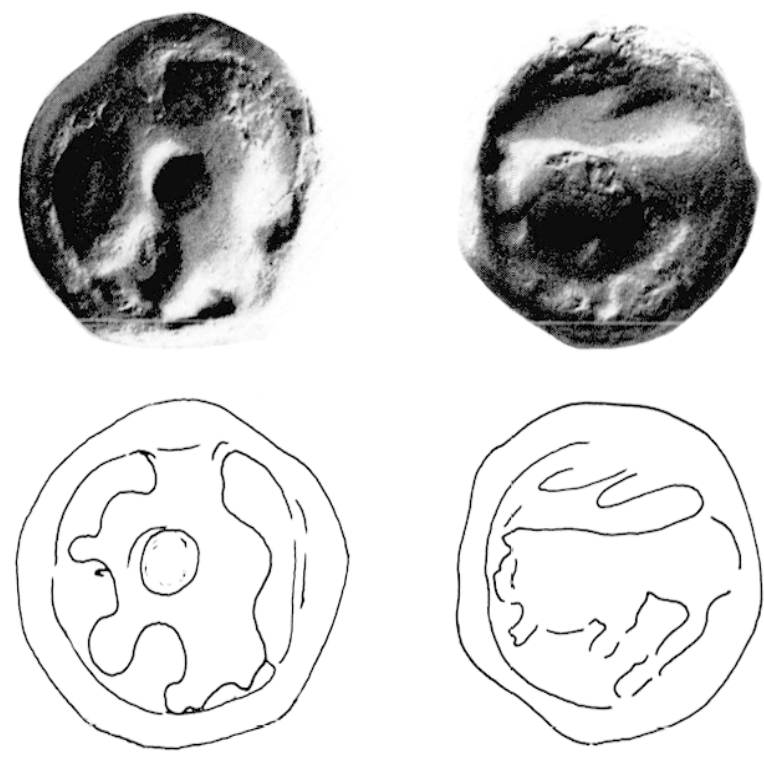

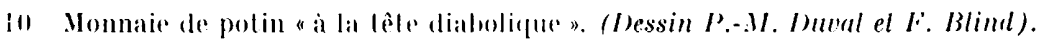

\section{Monnaies de bronze sans numéro d'invenlaire.}

Quatre pièces de bronze sont en très maurais élat. Lilles sont groupés dans un mème sachet sur lequel on peut lire: "Silvanectes, Carnutes et indéchiffrées" Les espèces de bronze se dégradent parfois très vite et quand les deux premières attributions ont été faites, ces monnaies étaient probablement mieux conservées. Leur aspect actucl ne nous parait permettre aucune identification.

18. L'une d'elles, toutefois, a pu itre considérée comme rarnute en raison de la typologie de la face tenant lieu de revers. (On y distingue. ('n effet, un quadrupede à droite surmonté d'une masse qui pourrait ètre un oiseau. La monnaie $\mathrm{BN} 6314^{44}$ correspond à cette représentation. Il faut toutefois signaler que la face qui tient lieu de droit paraît très différente sur ces deux espèces : l'exemplaire du Cabinet des Médailles porte un décor géométrique fait de fragments de guirlandes, de demi-cereles ot de petits cercles, alors que la monnaie du Vusée ('arnavalet, autant qur l'on an puisse juger, présente une masse qui semble correspondre au rendu d'un visage lummain. L'assimilation est done tris hypothétique. Poids : 4,89 gr ; module: : 1 i) $\mathrm{mm}$.

19. La deuxième monnaie semble itre un bronze frappe, elle pèse 2.84 g. son module est de $15 \mathrm{~mm}$.

20. La troisjeme, qui est un bronze frappé, porte des traces de dorure. Pour un poids de $2.91 \mathrm{~g}$. son module est de $15 \mathrm{~m}$ mm.

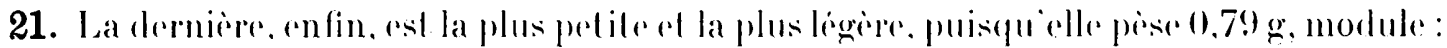
$12 \mathrm{~mm}$.

44 H. I: LA Torn, Alles..., pl. Nix. 
Les monnaies de bron\%e semblent avoir fait leur apparition all plus tard pendant la guerre des Gaules et leur circulation s' est poursuivie fort lardivement, au moins jusque sous Tibèrét.

La présence d'une monnair de la série dile "homotypique des Parisii " au Musée Carnavalet (ci-dessus $n^{0}$ 4) nous fournit l'occasion d'examiner de plus près ces espèces encore mal connues.

J.-B. Colbert de Beaulieu en a signalé quinze exemplaires dans son ètude : onze figurant au Cabinet des Médailles sous les nos 7801 à 7811 , trois appartenant à la collection Vian et un à la collection Deroc a Avignon. A cet ensemble, nous pouvons ajouter deux pièces, gràce à la publication récente de deux recueils, l'un dù à s. Schecers ${ }^{46}$, l'autre à K. Castelin ${ }^{47}$. Avec la monnaie du Musée liarnavalet, l'étude charactéroseopique a été effectuéc sur dix-huit espèces et a donné les résultats suivants:

\begin{tabular}{|c|c|}
\hline 7801 coins $\mathrm{D} 1 / \mathrm{K} 1$ & 7807 roins $\mathrm{D} 4 / \mathrm{K} 4$ \\
\hline 7802 coins $D 1 / R 1$ & 7808 coins $103 / 183$ \\
\hline $780: 3$ coins $102 / \mathrm{K} 2$ & 7809 coins $10: 3 / 133$ \\
\hline 804 coins $152 / \mathrm{R} 2$ & coins 1$) 4 / \mathrm{R} 4$ \\
\hline 05 coins $[92 / R 2$ & 811 ('oins I):3 \\
\hline
\end{tabular}

L'exemplaire $n^{0}$ j) de la collection Vian ${ }^{48}$ est de coins 1)2/R2. L'exemplaire $n^{0} 7$ de la même collection ${ }^{49}$ est de coins D)4/R4. La monnaie appartenant à la collection Deroc ${ }^{50}$ est de coins 1)4/R4. La pièce conservée au Musée de Zurich ${ }^{51}$ est de coins D3/R3. Celle qui figure dans la collection Danicourt ${ }^{52}$ est de coins $[$ :2/R:2, quant à celle du Yusée Carnavalet, elle a été frappée avec les coins 1$) \cdot \mathrm{R}$. Cn nombre d'exemplaires aussi limité ne permet pas de conclusions solides.

Cet examen nous fournit toutefois un renseignement intéressant : à un coin de droit correspond toujours le mème coin de revers, ce qui peut faire supposer un dispositif de solidarité des coins ayant servi à frapper ces pièces. Létude des exemplaires conservés au Cabinet des Védailles nous a permis de constater que, pour les espèces réalisées à l'aide des coins I)1/R1 et I)2/R2, à un point choisi au droit correspond toujours le même point au revers, ce qui est conforme à notre hypothèse. Pour les monnaies frappées à l'aide des coins I)3/R3, en revanche, il n'y a aucune correspondance.

Compte tenu de la technique de frappe à cette époque ${ }^{53}$. il faut raisemblablement attribuer à un hasard le phénomène concernant les deux premiers groupes. Les orientations

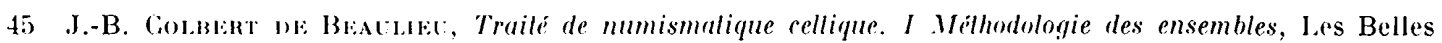
Lettres, Paris, 1973 , p. 236-239 et p. 3221-325.

46 Les monnaies gauloises de la collection A. Manicourt...

47 Kellische Münzen...

4\$ (iB, Parisii, p. 142, no 1.

49 Op. cil., p. $142, \mathrm{n}^{\circ} 8$.

50 Op. cil., p. 1.12, $\mathrm{n}^{\circ} 10$.

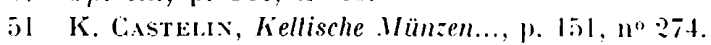

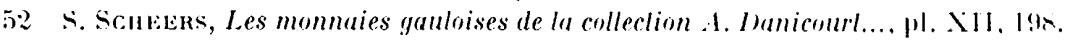

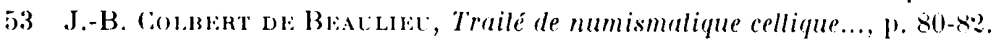


très différentes des coins observées sur les pièces de la série $[$ 3/R3 indiquent qu'il n'y avait pas de dispositif de solidarité.

Tout comme les monnaies des Parisii, ce numéraire élait done réalisé à l'aide d'un coin dormant et d'un coin mobile.

Une autre constatation peut ètre faite : quelques-unes de ces monnaies sont entaillées et cette pratique est assez courante pour des espèces d'or, mais la marque est normalement réalisée sur le revers, alors que, dans celle série, certains droils portent aussi une entaille. C'est le cas de la monnaie $n^{0} 4$ du Musée Carnavalet (entaillée au droit ot au revers), de BN 7801, BN 7803 (très légèrement entaillée au-dessus du nez), de BN 7801 (entaillée au droit et au revers), de BN 7805) (entaillée au droit et portant aussi une légère entaille au revers).

Par ailleurs, ces espèces se distinguent par leur métrologie : il s'agit de tiers de statère. Ce cas, s'il n'est pas unique dans la numismatique celtique (des tiers de statère existent aussi au -- $\mathrm{II}^{\mathrm{e}}$ siècle chez des peuples riverains de la Normandie), est néanmoins fort rare. Sa technologie et sa méthodologie font done de cette "série homotypique " un monnayage vraiment à part, qui se distingue nettement de celui des Parisii et mériterait une étude de caractère exhaustif.

Brigitte Fisciher

C.N.R.S. 